\title{
MAPS based vertex detector at STAR
}

\author{
E. Anderssen, X. Dong, L.C. Greiner, H.S. Matis, H.G. Ritter, J. Silber, T. \\ Stezelberger, X. Sun, M. Szelezniak', J.H. Thomas, C.Q. Vu, H.H. Wieman, Y. \\ Zhang, \\ Lawrence Berkeley National Laboratory \\ 1 Cyclotron Road, MS 70R0319, Berkeley, CA, USA \\ E-mail: maszelezniak@lbl.gov
}

\section{J. Kapitan}

Nuclear Physics Institute AS CR

25068 Rez/Prague, Czech Republic

E-mail: kapitanercf.rhic.bnl.gov

\section{S. Margetis, J. Bouchet}

Kent State University

Kent, OH, USA

E-mail: margetisestar.physics.kent.edu

\section{F. Videbaek}

Brookhaven National Laboratory

Upton, NY 11973, USA

E-mail: videbaekebn I.gov

\begin{abstract}
A new vertex detector upgrade that is being developed for the STAR experiment at RHIC is presented. This upgrade is named the Heavy Flavor Tracker, or HFT, and consists of three subdetectors that will extend the capabilities of the STAR detector in the heavy flavor domain. This paper focuses on the innermost sub-system, built with monolithic active pixel sensors. We present its requirements and geometry and address some of the challenging aspects of the detector design and construction, including sensors and batch testing, mechanical support design, and validation of the detector's cooling system.
\end{abstract}

19th International Workshop on Vertex Detectors - VERTEX 2010

Loch Lomond, Scotland, UK

June $06-112010$

\footnotetext{
$1 \quad$ Speaker
} 


\section{Introduction}

The Heavy Flavor Tracker is a tracking upgrade to the STAR detector at the RHIC collider [1] for precision measurements of the yields and spectra of particles containing heavy quarks. This upgrade will allow topological identification of mid rapidity Charm and Beauty mesons and baryons by reconstruction of their displaced decay vertices with unprecedented DCA (distance of closest approach) pointing resolution of $<30 \mu \mathrm{m}$.

The HFT consists of four concentric layers of silicon detectors grouped into three subsystems [2]. The outer two layers, at 22 and $14 \mathrm{~cm}$ radii, are built in double-sided and singlesided strip technologies, respectively. The two innermost layers at 2.5 and $8 \mathrm{~cm}$ average radii consist of Monolithic Active Pixel Sensors (MAPS) and constitute the PXL detector. The HFT will significantly enhance the pointing resolution of the Time Projection Chamber (TPC), which is the main STAR tracking detector. Precise vertex reconstruction in high track density events in $\mathrm{Au}-\mathrm{Au}$ collisions will be guaranteed due to graded pointing resolution from the outside detectors inwards, allowing the TPC pointing resolution of approximately $1 \mathrm{~mm}$ to be enhanced to the final resolution of $30 \mu \mathrm{m}$ of the PXL detector for particles with transverse momentum of $\sim 750 \mathrm{MeV} / \mathrm{c}$.

\section{PXL geometry and requirements}

Achieving the high accuracy impact parameter resolution (accuracy of pointing to the vertex) in the STAR environment requires a high granularity, thin detector with short integration time and good radiation tolerance. These requirements have to be balanced with mechanical stability and readout system requirements and constraints.

MAPS have been chosen for the PXL detector because their properties, when compared with other silicon detector technologies, best match the requirements for this detector. MAPS for the PXL detector will provide high granularity (approximately $20 \mu \mathrm{m}$ pixel pitch), relatively short integration time $(<200 \mu \mathrm{s})$, and thin sensors $(50 \mu \mathrm{m})$ achievable through commercially available thinning services.

The PXL detector is designed to have a thickness of approximately $0.37 \%$ of radiation length per layer in the tracking region. This radiation length includes the MAPS sensor, readout Kapton cable with aluminum traces, and a rigid carbon fiber support structure, which is designed to maintain the position of the pixels within a $20 \mu \mathrm{m}$ envelope. The operating temperature of the detector will be limited by an airflow based cooling system, the solution with minimal impact on the material budget.

The two-layer detector geometry is constructed with a set of 40 sensor ladders with ten 2 $\mathrm{cm} \times 2 \mathrm{~cm}$ sensors per ladder, leading to the total of approximately 400 million pixels. Ladders are mounted to 10 sectors, each sector features one ladder on the inside layer and three ladders at the outer layer. The coverage of the PXL detector is matched to that of the outer tracking detectors ( $|\eta| \leq 1,2 \pi$ in $\varphi$ ).

The described geometry of the detector and the chosen pixel pitch of approximately $20 \mu \mathrm{m}$ provide the pointing resolution of approximately $12 \oplus 19 \mathrm{GeV} / \mathrm{p} \cdot \mathrm{c} \mu \mathrm{m}$ for perpendicular tracks 
( $\mathrm{p}$ - particle momentum, $\mathrm{c}$ - speed of light in vacuum). It should be noted that the resolution of the detector will be limited by multiple-Coulomb scattering.

Due to the location of the detector, very close to the interaction point, the radiation dose to which the PXL detector will be exposed is estimated at 20 to $90 \mathrm{kRad}$ and up to $10^{13} \mathrm{neq} / \mathrm{cm}^{2}$ per year of operation, projected for maximum Au-Au RHIC II luminosity $\left(5-8 \times 10^{27} \mathrm{~cm}^{-2} \mathrm{~s}^{-1}\right)$. This radiation environment is compatible with MAPS radiation hardness, especially for sensors that utilize a depleted epitaxial layer [3].

\section{MAPS prototypes}

Sensors for the PXL detector are developed in collaboration with the CMOS-ILC group at IPHC, Strasbourg, which has a well established track record in MAPS design and development $[3,4]$. Development of the sensors for the PXL detector has gone through several stages. It started with small sensor prototypes featuring analog readout, which culminated in the installation of a 3-sensor telescope prototype in STAR, which tracked particles in the Au-Au collision environment [5]. Recently, this development has reached maturity with full-reticle size $\left(\sim 4 \mathrm{~cm}^{2}\right)$ devices with $30 \mu \mathrm{m}$ pixel pitch, column parallel readout and column level discriminators that allow for a binary readout of all pixels. Data is readout via four serial LowVoltage Differential Signaling (LVDS) outputs at $160 \mathrm{MHz}$, which translates to the sensor integration time of $640 \mu \mathrm{s}$. Laboratory tests indicate good performance with equivalent noise charge of approximately 15 electrons.

The next MAPS prototype dedicated to PXL will feature on-chip zero suppression, which will reduce data readout rates from sensors and, therefore, shorten the integration time to $<200$ $\mu$. This device will be the basis of the full sensor production for the PXL detector.

Development of MAPS for STAR benefits from the on-going development at IPHC and each of the aforementioned prototypes is based on other prototypes developed at IPHC for a range of applications. Some of the most important milestones for the PXL detector included Mimosa 8 [6] and Mimosa 26 [7] sensors, introducing column parallel readout with on-chip digitization and on-chip zero suppression, respectively.

The architecture of the final sensor for the PXL detector is based on the Mimosa 26 prototype. The on-chip zero suppression circuit will reduce the amount of data to be read out, which will allow to shorten the integration time together with limiting the number of fast LVDS outputs from 4 to 2, with respect to the current PXL sensor. The pixel size is expected to be reduced from the $30 \mu \mathrm{m}$ pitch used at present to approximately $20 \mu \mathrm{m}$. The pixel size is chosen to balance power consumption and radiation hardness, which have opposing requirements on the number of pixel per surface area. Optimization of power consumption at the level of $15 \%$ can also be achieved by lowering the power supply from the default $3.3 \mathrm{~V}$ to $3.0 \mathrm{~V}$. Laboratory tests indicate no degradation of performance when the sensor is operated at the lower voltage, however, additional tests are required, including detection efficiency studies on high energy particle test beams. 


\subsubsection{Probe testing thinned sensors}

Assembling a detector composed of ladders with $50 \mu \mathrm{m}$ thick sensors poses unique challenges in terms of handling and testing of silicon devices. Although the fabrication yield on sensors tested so far is above $90 \%$, testing of thinned sensors just before installing them on ladders will assure high yield in the ladder assembly process. The goal is to perform automated tests that allow for a qualitative analysis of sensors, including identification of dead or stuck pixels.

The construction plans for the PXL detector include building two full copies of the detector and a set of spare components. This approach will require testing of a few thousands of sensors, assuming reasonable fabrication and thinning yields. The former is typically expected to be at the level of $80 \%$ and the latter at $90 \%$.

Sensors designed for the STAR PXL detector feature in their pad rings additional pads dedicated to probe tests. These additional pads are associated with the bare minimum of IO signals that guarantee the required full testing capability. In this way, the high quality of the metal surface on the main pads is preserved for wire bonding.

For testing large numbers of sensors we have constructed a vacuum chuck shown in Figure 1 that will hold up to 20 thinned silicon dies for automated sensor tests on a standard automated probe station. The chuck has been built using a structure built with rapid prototyping combined with an aluminum vacuum plate. The loading of sensors onto the chuck is manual and aided by a vacuum on/off valve for each die. The chip alignment relies on ridges near each die that allow for precise positioning. Once all sensors are aligned, the test system will perform stepping through and testing all of the sensors without human intervention, and the accumulated offset errors are expected not to extend beyond all pad locations $\left(120 \times 80 \mu \mathrm{m}^{2}\right.$ pads). The design of the system has been finalized but full test results are not available at the time of this writing.

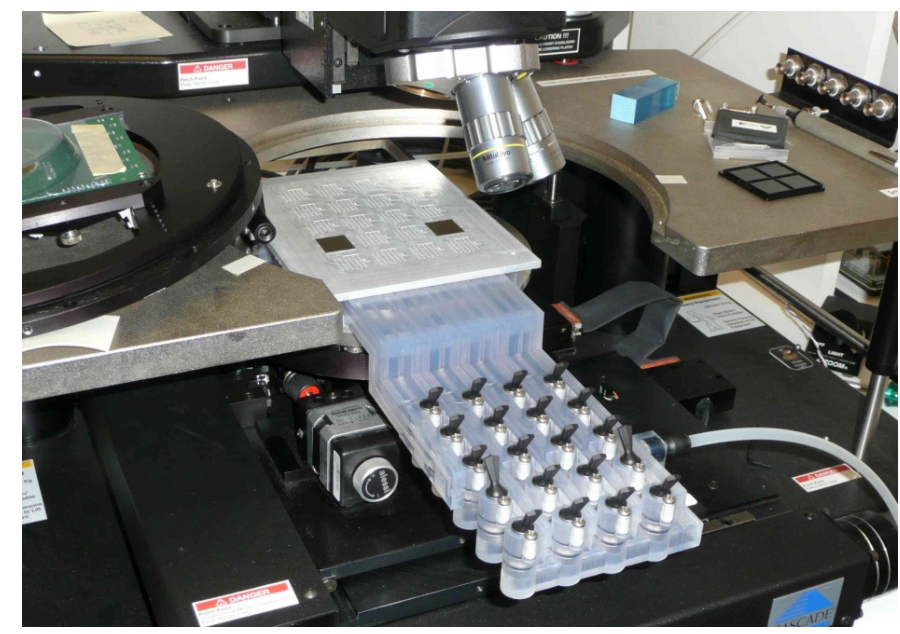

Figure 1: Overview of the vacuum chuck for testing $50 \mu \mathrm{m}$ thin MAPS prototypes before they are mounted onto ladder structures. Each die aligns to two ridges on the vacuum plate and each die location has an independent vacuum valve. 


\section{PXL readout system}

The PXL detector readout system is highly parallel and consists of ten independent units, each serving one sector of the detector. Each sector is connected with $2 \mathrm{~m}$ of fine twisted-pair wires to a mass termination board, which provides buffering for signals going to/from each ladder. The buffered signals are routed on a much more robust twisted-pair cable to the readout motherboard. In addition to buffering, the mass termination board provides independent latch up protected power supplies for each ladder. The heart of the readout system is the motherboard attached to a Virtex-5 FPGA development board, which groups data into events formed in response to STAR triggers and sends data to a readout PC via an optical link.

Buffering boards and power regulators will be located in the STAR experiment inside of the TPC in a moderately high radiation area. The readout boards (Virtex-5) will be located outside of the TPC, where they should be safe from single event upsets. A more detailed description of this setup can be found in [8].

\section{Mechanical support}

A large part of the detector development effort is dedicated to designing and building a mechanical support structure. The main requirements driving this development include the required complete spatial mapping and detector mechanical stability within a $20 \mu \mathrm{m}$ window, compatibility with an air-flow based cooling system, and rapid detector removal and installation. The first design goal, complete spatial mapping, is important to achieve physics results in a timely fashion. It is required that locations of all pixels with respect to each other are known to within $20 \mu \mathrm{m}$ at the installation time and these positions are maintained through the lifetime of the detector. The last requirement aims at replacing the PXL detector with a new, fully functioning copy of the detector within one day ${ }^{1}$ in case of a catastrophic failure or diminished efficiency due to high radiation.

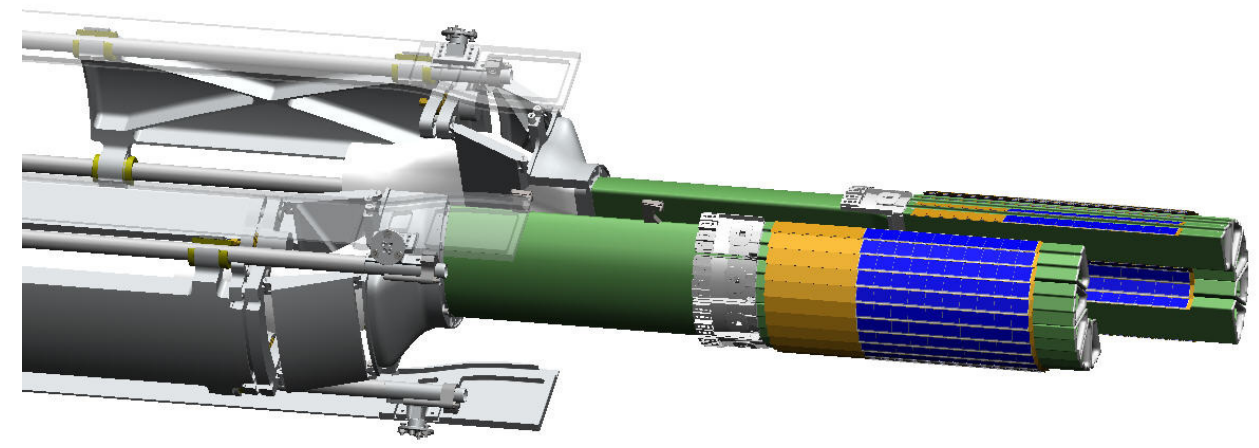

Figure 2: Overview of the PXL detector mechanics showing detector barrel, support structures and insertion parts. The right half of the detector is shown slightly retracted.

An overview of the pixel detector is shown in Figure 2. PXL consists of two concentric barrels of detector ladders, which are approximately $25 \mathrm{~cm}$ long. The inner barrel has a radius of

\footnotetext{
${ }^{1}$ The one day time period is a typical time that RHIC has access times while the machine is in operation.
} 
$2.5 \mathrm{~cm}$ and the outer barrel has an $8 \mathrm{~cm}$ radius. The barrels separate into two halves for assembly and removal. In the installed location both barrel halves are supported by their own three-point precision kinematic mounts located at one end.

A well controlled method for installation of the pixel detector has been developed with emphasis on ease of operation and avoidance of detector risk. The insertion mechanism has been designed to guide the detector around the beam pipe. The insertion device rides on rails and uses a cam guide to close the detector halves around the beam pipe and guide the detector into fixed kinematic mounts that define the final operating position of the PXL detector. A twin mechanical setup will be used for spatial mapping of the detector in its internal coordinate system using a visual or touch coordinate measuring machine. A fully mapped detector can then be deployed in the STAR experiment using the insertion mechanism with the mapping preserved.

\section{Air cooling system}

The operating detector will dissipate about $350 \mathrm{~W}$ in the low mass region; the silicon sensors generate $170 \mathrm{~mW} / \mathrm{cm}^{2}$ and the readout buffers for each ladder generate approximately $2 \mathrm{~W}$. The large difference in coefficient of thermal expansion (CTE) between silicon sensors and kapton of the readout cable is a potential source of thermal induced deformation. Simulations indicate that deformations can reach $10 \mu \mathrm{m}$ at a $20^{\circ} \mathrm{C}$ temperature difference. An efficient cooling system is necessary to guarantee the sensor performance and the mechanical stability of the ladders.

Cooling of the PXL detector is provided by air flowing in from one end between the two barrel surfaces and returning in the opposite direction over the outer barrel surface and along the inner barrel surface next to the beam pipe.

The efficiency of this cooling system has been simulated using computational fluid dynamics and measured with a full size detector mockup deployed in a dedicated wind tunnel.

At the time the detector mockup was constructed, full size silicon sensors dissipating the appropriate amount of power were not available. Mockup ladder structures featured kapton cables with copper traces forming heaters, which allowed the system to dissipate the expected amount of power in the detector. Each ladder was equipped with 6 thermistors for temperature monitoring purposes. One of the sectors was equipped with 10 dummy silicon chips per ladder with platinum heater traces vapor deposited on top of the silicon. The temperature of the setup was measured using the aforementioned thermistors and a thermal camera. Airflow was provided by an industrial type air pump and air speed was measured with an air-velocity probe inserted between the wind tunnel and the outer detector layer.

The measured cooling efficiency is summarized in Figure 3, which shows the temperature increase at different locations of the ladder as a function of airflow velocity. Ambient temperature and the cooling air temperature in this test were at $27^{\circ} \mathrm{C}$. The measurement results show the highest temperature in the detector is the middle of each ladder and it is up to $12{ }^{\circ} \mathrm{C}$ higher than the temperature of the cooling air. The measurements with the mockup detector roughly confirm simulation results. 


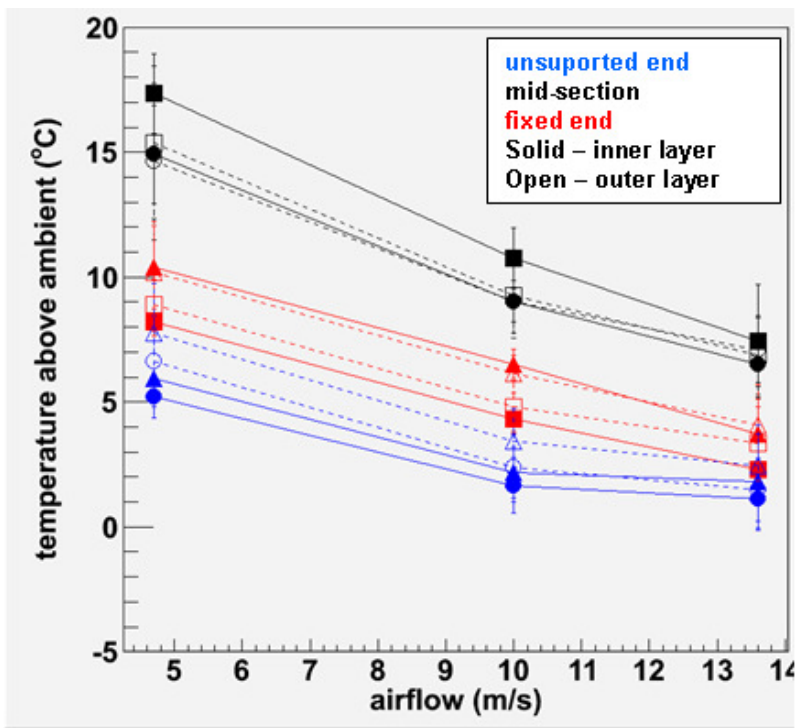

Figure 3: Cooling efficiency measured with the PXL detector mockup dissipating $~ 350 \mathrm{~W}$ at various airflow speeds. Temperature measurements are averaged for all thermistors at the same location on each ladder and referenced to the input air temperature.

The presented setup was also used for studying the mechanical stability and quantifying air-flow-induced vibrations. Results indicate that for air speeds up to approximately $10 \mathrm{~m} / \mathrm{s}$ the mechanical stability is within the required stability windows, but detailed analysis of the vibrations in this system is beyond the scope of this paper.

\section{Summary}

The STAR Heavy Flavor Tracker upgrade group is working to extend the capabilities of the STAR detector in the heavy flavor domain by providing a tracking system that will allow for very high resolution vertex measurements. In the center of this upgrade is a MAPS based, 2layer PXL detector. A well established sensor development plan in conjunction with IPHC is on track to deliver full reticule sized sensors featuring zero suppression and digital readout. Tools and techniques for batch testing of thinned sensors have been developed and are being tested. Advanced prototypes of readout electronics have been built and validated. Development of the detector's mechanical support, including concept designs for installation and insertion, is well advanced with first prototypes being produced. Prototype tooling for sector/ladder production has been developed and successfully used for prototype detector mockup assembly. The mockup setup allowed for a successful validation of the PXL detector cooling system. Tests of ladder prototypes with 10 full-size sensors are on-going with the goal of building a complete prototype detector, combining mechanical support, complete sectors, and readout electronics.

\section{Acknowledgments}

We gratefully acknowledge Marc Winter and the IPHC group for the development of the PXL detector sensors. This work received support in part from the US Department of Energy Contract no. DE-AC02-05CH11231, Office of Nuclear Physics. 


\section{References}

[1] J. Adams, et al. Experimental and theoretical challenges in the search for the quark-gluon plasma: The STAR Collaboration's critical assessment of the evidence from RHIC collisions, Nuclear Physics A, Volume 757, Issues 1-2, First Three Years of Operation of RHIC, 8 August 2005, Pages 102-183.

[2] C. Chasman et al., A Heavy Flavor Tracker for STAR, 2008, LBNL/PUB-5509-2008.

[3] M.Winter, Achievements and perspectives of CMOS pixel sensors for charged particle tracking, Nucl. Instr. and Meth. A, 2010, doi:10.1016/j.nima.2010.02.192.

[4] G. Gaycken, et al., Monolithic active pixel sensors for fast and high resolution vertex detectors, Nucl. Instr. and Meth. A, 2006, doi:10.1016/j.nima.2005.11.233.

[5] L.C. Greiner, et al., STAR Vertex Detector Upgrade Development, PoS (Vertex 2007) 041.

[6] Y. Degerli, et al., A fast monolithic active pixel sensor with pixel-level reset noise suppression and binary outputs for charged particle detection, IEEE Trans. Nucl. Sci, 52, 2005, pp. 3186-3193.

[7] C. Hu-Guo et al., First reticule size MAPS with digital output and integrated zero suppression for the EUDET-JRA1 beam telescope, Nucl. Instr. and Meth. A, 2010,DOI:10.1016/j.nima.2010.03.043.

[8] M. Szelezniak, et al., CMOS pixel vertex detector for STAR, PoS (Vertex 2008) 032. 